\title{
MIGRACIÓN, SALUD Y GÉNERO: ABORDAJES DE PROVEEDORES DE SALUD EN LA ATENCIÓN DE MUJERES MIGRANTES BOLIVIANAS EN LA PROVINCIA DE MENDOZA, ARGENTINA
}

Immigration, health and gender: health care team's approaches in the care of Bolivian migrant women in the province of Mendoza, Argentina

\author{
Lila Aizenberg*
}

Gabriela Maure $^{* *}$

\begin{abstract}
Resumen. Si bien la articulación entre migración internacional y salud ha ido en aumento en la literatura, son incipientes aún los estudios interesados en analizar cómo los sistemas sanitarios responden a la atención de mujeres migrantes. Este artículo analiza las opiniones de proveedores de salud en relación a la atención ofrecida a mujeres migrantes bolivianas en la provincia de Mendoza, Argentina. Se trata de un estudio exploratorio y descriptivo con base en entrevistas en profundidad y grupos focales realizadas a personal sanitario. El trabajo muestra distintos tipos de abordajes en la atención de las mujeres migrantes que varían entre aquellas donde se reproducen prácticas de disciplinamiento y control hacia otras que se recuperan las condiciones objetivas e históricas que las mujeres desarrollan sus procesos de salud y los factores culturales, laborales y de género que las atraviesan en el contexto migratorio.
\end{abstract}

Palabras claves: migración, salud, género, interculturalidad, Argentina.

\begin{abstract}
Even though the health-care problem has been analyzed by the field of study of migration, there are still few studies that address how health systems respond to the health care of migrant women. This article analyzes health care team's opinions on Bolivian migrant women in the province of Mendoza, Argentina. This is an exploratory and descriptive study based on in-depth interviews and focus groups on health professionals. The study shows different types of approaches that vary between those where practices of discipline and control are reproduced towards others where the socio-historical conditions behind women's health processes as well as the cultural, labour and gender factors that affected them in the migratory context are taking into account.
\end{abstract}

Keywords: immigration, healthcare, gender, interculturality, Argentina.

\footnotetext{
* Investigadora del Conicet-Ciecs, Universidad Nacional de Córdoba. Córdoba, Argentina.

** Becaria doctoral del CONICET, Universidad Nacional de Cuyo. Mendoza, Argentina.
} 


\section{Introducción}

Si bien desde hace más de treinta años la articulación entre salud y migración viene siendo abordada desde las ciencias sociales ${ }^{1}$, todavía resulta incipiente el conocimiento existente respecto a cómo el sistema biomédico responde a la atención de las mujeres migrantes. América Latina fue la primera región del mundo en desarrollo en alcanzar el record de paridad entre el número de varones y mujeres migrantes como producto de la complementariedad entre la situación de los mercados de trabajo de los países, la demanda laboral en actividades de servicios, los efectos de las redes sociales y las modalidades de la reunificación familiar ${ }^{2}$. A ello se suman las oportunidades laborales generadas en algunos países latinoamericanos en donde han surgido nichos específicos que atraen flujos laborales con calificaciones y características determinadas, dando lugar a lo que Saskia Sassen ${ }^{3}$ ha señalado como la conexión entre las necesidades de las ciudades globales de contar con mano de obra a bajo costo y la feminidad de la migración. Sin dudas, el alto grado de feminización que ha venido caracterizando a la población migrante latinoamericana, donde se encuentra una importante cantidad de mujeres migrantes en edad reproductiva, impone al sistema de salud de los países receptores el desafío de garantizar las condiciones que puedan satisfacer a dicha demanda ${ }^{4}$. Hace algunos años, las ciencias sociales aplicadas al campo migratorio han planteado la necesidad de abordar la interseccionalidad de las dimensiones de género, etnicidad, clase social y origen nacional en los resultados experimentados por las personas en contextos migratorios ${ }^{5}$. Los distintos trabajos que han analizado el impacto de la migración sobre la salud de las mujeres en América Latina han colocado a la migración como un factor de riesgo para la salud, mostrando que la confluencia del género, la etnia, la clase social, las condiciones laborales, la nacionalidad, la falta de perspectiva intercultural y la indocumentación pueden conducir a las más extremas violaciones de derechos humanos, incluyendo abusos sexuales, deterioro de la salud reproductiva y amenaza a la integridad física ${ }^{6}$.

A pesar de que se ha puesto en evidencia la feminización como una característica saliente de los actuales procesos migratorios y a la salud como un aspecto clave de las experiencias de quienes migran, todavía no se ha analizado

1 AIZENBERG, Lila, RODRIGUEZ, María Laura, CARBONETTI, Adrián. Percepciones de los equipos de salud en torno a las mujeres migrantes bolivianas y peruanas en la ciudad de Córdoba, p. 68.

2 MARTÍNEZ PIZARRO, Jorge, REBOIRAS-FINARD, Leonardo. Migración, derechos humanos y salud sexual y reproductiva: delicada ecuación en las fronteras, p. 9.

3 SASSEN, Saskia. Globalization and its Discontents: Essays on the New Mobility of People and Money.

4 CERRUTTI, Marcela. Salud y migración internacional: mujeres bolivianas en la Argentina.

5 ANTHIAS, Floya. Belongings in a globalising and unequal world: rethinking translocations, p. 17.

6 MARTÍNEZ PIZARRO, REBOIRA-FINARD, op. cit., p. 10. 
en profundidad cómo responden los sistemas de salud biomédicos a las mujeres migrantes desde una perspectiva de género. Más específicamente, no ha sido suficientemente problematizado la forma en que opera el sistema de salud biomédico en la atención de mujeres migrantes a partir de la identificación de la mujer boliviana y de las prácticas que se imparten a partir dicha identificación. En este trabajo nos interesa comprender la producción discursiva, los modos y procedimientos implicados en las miradas y recomendaciones de los proveedores de salud, en tanto instancias co-constitutivas emergentes de un mismo proceso. Dicho proceso nos lleva a una creciente preocupación que instituye como problema la tarea de "administrar la vida" de las mujeres migrantes a través de la gestión del sistema biomédico ${ }^{7}$. Como señala Esteban", la práctica médicosanitarista ha estado atravesada por "sesgos de género" donde la perpetuación de ópticas deterministas y biologicistas de la realidad de las mujeres suelen permanecer invisibles para los propios profesionales. Aquí, las mujeres han sido contempladas como meras reproductoras biológicas donde la maternidad se ha pensado dependiente de la naturaleza, a partir de la separación absoluta entre lo doméstico y lo público, lo productivo y lo reproductivo. Si bien el mayor protagonismo que ha tenido la "mujer" como actor social relevante y en la esfera productiva en particular ha llevado a rediscutir ciertos roles y estereotipos de género, esto no necesariamente llevó a un cuestionamiento profundo de la tradicional división sexual y social del trabajo. En este sentido, el espacio "reproductivo"/"doméstico" ha estado atravesado por una "ideología de la domesticidad" ${ }^{\prime \prime}$ que ha definido al hogar como el lugar "ideal" de las mujeres. Aunque las representaciones de género han sufrido modificaciones, aquella ideología no ha perdido vigencia en el imaginario social en general y en el sistema sanitario en particular. Este trabajo acompaña a la crítica feminista en el esfuerzo por generar una revisión y desnaturalización del análisis clásico de la reproducción y de la sexualidad, resaltando el carácter dinámico de los procesos reproductivos/productivos ${ }^{10}$ así como el control social que se ejerce sobre ellos. Sostenemos que la forma en que el modelo biomédico interpela a las mujeres migrantes bolivianas, requiere una mirada profunda sobre la manera en que opera el sistema biomédico sustentando la construcción de

FOUCAULT, Michel. Vigilar y Castigar: Nacimiento de la prisión.

8 ESTEBAN, María José. El Estudio de la Salud y el Género: Las Ventajas de un Enfoque Antropológico y Feminista, p. 13.

9 Para profundizar ver: NASH, Mary. Identidad cultural de género, discurso de la domesticidad y la definición del trabajo de las mujeres en la España del siglo XIX.

${ }^{10}$ En términos conceptuales, el trabajo productivo hace referencia al destinado a la producción de bienes y servicios para ser intercambiados en el mercado a cambio de una retribución; a diferencia del trabajo de reproducción, que se realiza para la familia, sin obtener retribución y fuera del mercado laboral (BENERIA, Lourdes. Trabajo productivo/reproductivo, pobreza y políticas de conciliación). Al realizarse fuera del mercado de trabajo, este último se invisibiliza y no es reconocido como "trabajo". 
roles de género, la valoración de sus prácticas y las recomendaciones que se hacen respecto a los comportamientos que ellas deben llevar adelante para transformase en un determinado tipo de paciente y de mujer acorde a un modelo de salud deseado. En particular, entendemos que este enfoque se muestra especialmente adecuado para comprender los mecanismos que desde los sistemas de salud condicionan el acceso a los sistemas sanitarios de las mujeres así como para pensar un abordaje que incorpore una perspectiva intercultural en la atención de las mujeres migrantes. En este sentido, este trabajo acompaña aquellas miradas sobre la salud intercultural que enfatizan la importancia de dar cuenta en profundidad cómo las condiciones materiales de vida y especialmente el trabajo productivo-reproductivo que realizan las mujeres, dan forma a los procesos de salud-enfermedad-atención y a las maneras en que dichos procesos son transitados.

\section{Aspectos metodológicos}

La posibilidad de conocer cómo el sistema de salud opera sobre la salud de las mujeres migrantes bolivianas en Mendoza, Argentina, cobra particular relevancia tomando en cuenta las particularidades de la problemática de la migración en la actualidad. A partir de 1980 Argentina pasó a convertirse en un lugar de destino de los migrantes sudamericanos ${ }^{11}$. Según el Censo Nacional de Población y Vivienda de 2010, la provincia de Mendoza recibió al 8\% del total de los migrantes bolivianos que llegaron al país, los cuales constituyen el principal grupo migratorio en la provincia. La población boliviana en Mendoza proviene fundamentalmente de áreas rurales andinas de Bolivia, principalmente de Departamentos como La Paz, Potosí y Cochabamba, los cuales se autodefinen desde su origen étnico como quechuas y aymaras. En la actualidad, los desplazamientos territoriales hacia Mendoza mantienen pleno vigor y constituyen prácticas orientadas a mantener una mayor continuidad en la asalarización y a incrementar los ingresos anuales. Una de las regiones elegidas por las y los migrantes bolivianos en Mendoza es la zona del Valle de Uco (departamentos de San Carlos, Tupungato y Tunuyán). Esta región, ubicada al centro-oeste de la provincia, se caracteriza por ser una zona de gran desarrollo agrícola vitivinícola, de frutos y hortalizas que requiere de una importante mano de obra durante los períodos de cosechas, la cual es desarrollada por toda la familia campesina donde hombres y mujeres de toda edad participan en uno u otro momento de su vida en labores agropecuarias ${ }^{12}$. El sector agrícola se caracteriza por actividades manuales con jornadas laborales extensas y prácticas que muchas veces implican una importante carga física.

\footnotetext{
${ }^{11}$ PANTELIDES, Edith, MORENO, Martín. Situación de la población en Argentina.

12 BALÁN, Jorge. La economía doméstica y las diferencias entre los sexos en las migraciones internacionales: un estudio sobre el caso de los bolivianos en Argentina, p. 269.
} 
La situación de transitoriedad del trabajo agrícola sumada a la precariedad del empleo, coloca a migrantes bolivianos en una situación de alto nivel de vulnerabilidad debido a su invisibilidad social y a su mayor desprotección legal respecto a otros trabajadores. En su gran mayoría, los migrantes sudamericanos en Argentina se enfrentan con importantes obstáculos para el cuidado de su salud debido a la combinación de distintos determinantes sociales como el bajo nivel educativo, las condiciones desfavorables de vivienda, el escaso acceso a los servicios de infraestructura básica y las condiciones laborales insalubres o precarias, entre otros ${ }^{13}$. En el caso específico de las mujeres, esta vulnerabilidad se ve potenciada en tanto éstas deben dividirse entre las tareas productivas y reproductivas, principalmente vinculadas a roles tradicionales que históricamente las han colocado como responsables "naturales" del cuidado de los otros. En este sentido, además de la falta de cobertura de salud, producto de la precariedad laboral que comparten con sus pares varones, las mujeres migrantes se enfrentan con una multiplicidad de barreras vinculadas al cuidado de su salud, especialmente durante la edad reproductiva, que abarcan dimensiones de género, de clase social, culturales e institucionales que enfrentan en las instituciones públicas de salud a las que acuden para su propia atención y la de sus hijos ${ }^{14}$.

Este estudio se llevó a cabo entre marzo y julio del 2016. La estrategia metodológica utilizada fue cualitativa. La investigación consistió en un estudio exploratorio y descriptivo con base en entrevistas a profundidad a un agente sanitario perteneciente al primer nivel de atención y a grupos focales dirigidos a un equipo interdisciplinario compuesto por 1 psiquiatra y 1 psicóloga y 2 trabajadoras sociales del tercer nivel de atención del principal hospital de referencia. Durante el trabajo de campo, las entrevistas al agente sanitario se realizaron durante sus visitas a un asentamiento de viviendas que concentra mayoritariamente población de origen boliviano. Ubicado al costado de una ruta nacional en una zona rural agrícola, el asentamiento se creó hace alrededor de quince años al compás de la llegada de familias bolivianas. Aunque la cantidad de hogares fue creciendo significativamente desde su inicio, esto no estuvo acompañado de mejoras a nivel de infraestructura, lo que lleva a que la población se encuentre viviendo en una situación de alta precariedad habitacional y social que repercute fuertemente en su calidad de vida y estado de salud. Las entrevistas al resto del personal sanitario se realizaron dentro de sus lugares de trabajo. En ambos casos las entrevistas rescataron sus percepciones respecto a la población migrante boliviana así como a las barreras y facilitadores que existen en el acceso y el uso de las mujeres migrantes a los

\footnotetext{
${ }^{13}$ CERRUTTI, Marcela. Diagnóstico de las poblaciones de inmigrantes en la Argentina.

${ }^{14}$ AIZENBERG, RODRIGUEZ, CARBONETTI, op. cit., p. 75.
} 
servicios sanitarios públicos de la zona geográfica seleccionada. Según normas y acuerdos éticos nacionales e internacionales, se informó a los entrevistados la finalidad de la entrevista y el carácter voluntario de su participación, así como el carácter anónimo de la entrevista y de la institución. Las entrevistas fueron realizadas por investigadores sociales, los cuales se presentaron como externos e independientes a las instituciones de salud. Todas fueron grabadas con consentimiento de la persona entrevistada y posteriormente transcritas para su posterior procesamiento y análisis. La codificación de las entrevistas y el análisis fueron realizados manualmente, considerando los principales tópicos con los cuales fue construido el problema de análisis.

\section{El proceso de construcción de un abordaje intercultural en la atención de las mujeres migrantes}

En esta sección se analizan y describen las narrativas de un equipo de salud interdisciplinario del tercer nivel de atención ${ }^{15}$ compuesto por psicólogos, psiquiatras y trabajadores sociales del Municipio de Tupungato donde se pone en evidencia las estrategias que los profesionales desarrollan para construir un marco explicativo de relaciones interculturales donde se incorpora al otro (a) y a sus especificidades históricas, culturales, sociales, económicas y subjetivas incluidas las desigualdades de género que subyacen a sus prácticas en salud. Esta sección acompaña a los estudios sobre salud intercultural que han enfatizado la importancia de incorporar las condiciones socio-políticas, económicas y vinculadas a las expectativas sociales de género que explican el acceso de las poblaciones a los servicios sanitarios así como el marco de relaciones interculturales entre usuarias y proveedores ${ }^{16}$. Abordar la particularidad de cómo el sistema de salud opera sobre las mujeres migrantes bolivianas requiere primeramente comprender el carácter etnocentrista del sistema médico y el patriarcado como formas que refuerzan al biologicismo desde sesgos sexistas, étnicos y de clase. Desde principios del siglo XX, el cuerpo de las mujeres en los sistemas sanitarios estuvo atravesado por la perspectiva higienista en salud, la cual ha privilegiado su función reproductiva en tanto madres y como responsables por la alimentación, la higiene y el bienestar de los otros ${ }^{17}$. Cuando el dominio genérico y biologicista del sistema sanitario se entrecruza con la migración y la etnia, mujeres migrantes bolivianas son juzgadas por sus comportamientos a partir de una mirada estereotipada que interpreta la figura

\footnotetext{
${ }^{15}$ El tercer nivel de atención se reserva para la atención de problemas poco prevalentes, se refiere a la atención de enfermedades complejas que requieren procedimientos especializados y de alta tecnología.

${ }^{16}$ AYALA, Encarnación. La mujer en la perspectiva intercultural; AIZENBERG, Lila. Hacía una aproximación crítica a la salud intercultural.

17 FOUCAULT, op. cit.
} 
de la migrante boliviana como desinteresada de la crianza de sus hijos y de las tareas domésticas. En Argentina, la construcción de la figura migrante ha estado atravesada por imaginarios y estereotipos sociales que refieren a la etnicización "en clave nacional" y que en el caso de la mujer boliviana están asociados a la sumisión, la pasividad y docilidad ${ }^{18}$ respecto al varón y a las funciones reproductivas. Como se observa en la cita siguiente a través del registro que realiza el equipo interdisciplinario, esta construcción atraviesa también el espacio sanitario, el cual responde a través de mecanismos sancionatorios hacia aquellas mujeres que se dividen entre el mundo doméstico y el público.

Se las cuestiona... Es muy común en la población dejar al hijo para ir a trabajar... o llevarlos y tenerlos así en canastos... o dejarlo a la crianza de otros mientras ella va a trabajar o que se va con los familiares, con los hijos más grandes. Y ahí entran en conflicto con las instituciones porque ellas como mujeres trabajan, no son solo madres y entonces el prejuicio de las instituciones es que ellas tienen que ser madres (psiquiatra, grupo focal, mayo, 2016).

Insertos en el sistema biomédico pero como parte de un saber subestimado, psicólogos, psiquiatras y trabajadores sociales reconocen las limitaciones que tiene para llevar adelante su práctica cotidiana. Muchas de las características del sistema biomédico se enmarcan dentro de los elementos específicos de la biomedicina, como su paradigma positivista de pretensiones universales intolerante hacia otros modelos explicativos, su carácter eminentemente reduccionista y biologicista en donde lo psicológico y lo social son meros epifenómenos que ocultan la "verdadera" realidad ${ }^{19}$. Todo ello construye una estructura y una serie de prácticas donde los migrantes y su cultura entran en las explicaciones de los fracasos y las dificultades de la atención. En nuestro caso de estudio, el encuentro con la población migrante Ileva al equipo interdisciplinario a desarrollar una mirada introspectiva respecto a las limitaciones que se generan pero producto del rol que ocupan dentro del modelo biomédico para evaluar, vigilar y disciplinar a la mujer en un rol materno pretendido. Como se observa abajo, esta limitación se vuelve conflictiva en tanto no sólo se los separa de su función reflexiva "de escucha" y "evaluación" sino que se los aparta de las propias mujeres que evitan estos espacios ante la mirada controladora y sancionadora del personal de salud.

Siempre vienen para evaluación del "rol materno" al espacio de salud mental y lo que se defendía desde ahí era que no se puede brindar un espacio de escucha y de evaluación. La idea es trabajar desde otro lugar pero es muy difícil porque se da ahí un juego del Estado, del rol del

\footnotetext{
${ }_{18}$ BAEZA, Brígida. La memoria migrante y la escucha de los silencios en la experiencia del parto de mujeres migrantes bolivianas en Comodoro Rivadavia (Chubut, Argentina).

${ }^{19}$ KLEINMAN, Arthur. Writing at the margin. Discourse between anthropology and medicine.
} 
Estado de controlador que dice, "no sos buena madre, está el hogar, etc." (trabajador social, grupo focal, mayo, 2016).

El desencuentro entre el personal de salud y las migrantes no sólo se explica por el carácter disciplinador del sistema biomédico sino también por la forma en que cada grupo social construye sus nociones de cuerpo y de salud. Por un lado, la medicina formula el cuerpo humano y la enfermedad de una manera culturalmente específica en un proceso particular de construcción cultural de sus objetos ${ }^{20}$. La construcción cultural de la enfermedad en el modelo biomédico se configura exclusivamente desde la perspectiva biologicista, donde aspectos sociales y laborales quedan invisibilizados ${ }^{21}$. Sin embargo, como se evidencia en la cita siguiente, para las mujeres bolivianas el binomio cuerpo-salud no puede estar desligado de la vida laboral-productiva que las atraviesa en el contexto migratorio. En las sociedades andinas de tipo comunitario, el cuerpo y la salud no existen como elemento de individuación sino como parte de un conjunto de factores sociales, afectivos, ambientales, espirituales que determinan la armonía interna y externa, individual y comunitaria, en donde las condiciones donde se lleva adelante la vida laboral cobra una particular relevancia ${ }^{22}$.

La vida está organizada en función del trabajo y como se desplazan... de hecho claro, dónde viven depende de donde estén trabajando... El trabajo es como como que muy, muy, organizador así la vida donde están, qué hacen, es el trabajo... (psicóloga, grupo focal, mayo, 2016).

La identificación de la vida productiva como parte central de cotidianeidad de las bolivianas abre en los profesionales interesantes reflexiones sobre los roles socialmente esperados para estas mujeres y las desigualdades de género que padecen en el contexto migratorio. Dentro de los movimientos migratorios, la participación de las mujeres en los procesos productivos fue subestimada tanto por la literatura académica como por los propios actores que interactúan con las migrantes -como es el caso de los profesionales sanitarios-, producto del atravesamiento de la "ideología de la domesticidad". En este proceso, el modelo biomédico ha colocado a las mujeres en roles que, presentados bajo una supuesta "naturalidad", ha escondido desigualdad sociales, de género y étnicas llevando a reproducir estereotipos culturales de mujeres pasivas, acompañantes del varón y reproductoras del espacio doméstico como parte de un modelo eminentemente biológico y desligado del contexto social que la rodea.

${ }^{20}$ GOOD, Bayron. Medicina, Racionalidad y experiencia: una perspectiva antropológica.

${ }^{21}$ CARAMÉS GARCÍA, Mayte. Proceso socializador en ciencias de la salud. Caracterización crítica del modelo hegemónico vigente.

${ }^{22}$ RODRIGUEZ, Lilia. Factores Sociales y Culturales Determinantes en Salud: La Cultura como una Fuerza para Incidir en Cambios en Políticas de Salud Sexual y Reproductiva. 
Para ella (paciente boliviana) dejar al hijo no tenía que ver con la dejadez de no estar presente en la crianza sino que la crianza es un espacio más de la vida que ellas tienen que cubrir además del trabajo, o sea no es percibido esta cuestión como desinteresada por los hijos... sino como parte de las cosas que tiene que hacer... (trabajadora social, grupo focal, mayo, 2016).

En el proceso migratorio las tareas de cuidado en las poblaciones migrantes y la construcción social del rol materno está atravesada en muchos casos por cómo se van reconfigurándose los roles productivos y reproductivos. Para ellos, identificar las características que asume la vida cotidiana de las mujeres pone en cuestionamiento las representaciones hegemónicas de género, la transformación que asume el significado de la maternidad y las relaciones de pareja a partir de la experiencia migratoria. Así se reconoce que las familias deben adaptarse a una separación espacio-temporal o bien a arreglos de cuidado intrafamiliares y sociales reconfigurando roles de cuidado a partir del apoyo que reciben de las redes sociales, desde lo que Parella menciona como una lógica de un flujo continuo y no tanto de una radical división de experiencias separadas entre dos mundos ${ }^{23}$. También, la inserción laboral dentro del proceso migratorio produce a los ojos de los profesionales sanitarios situaciones paradójicas para las migrantes donde si bien se les reconoce la apertura de nuevos espacios de autonomía obtenidos a partir de su inserción en la vida pública-laboral, también se las identifican dentro de vínculos de dependencia y atravesados por situaciones de violencia de género al interior de su vida privada.

Ellas se definen no como amas de casa, "soy obrero rural o empleada rural", decían..., las mujeres son muy fuertes dentro de la familia boliviana pero desvalorizadas en la relación de pareja donde aparece esta cuestión de la violencia de género donde por los celos o por el control que establece el hombre sobre la mujer ella es ubicada en el lugar de menor valor (psiquiatra, grupo focal, mayo, 2016).

Reconocer cómo opera el sistema biomédico en la construcción y reproducción de desigualdades de género así como los itinerarios laborales que desarrollan las mujeres en el contexto migratorio abre interesantes posibilidades para romper con la lógica vertical y autoritaria del modelo médico hegemónico y para pensar relaciones desde un modelo que incorpore verdaderamente al otro(a), las trayectorias particulares de este flujo migratorio y sus dificultades específicas de acceso a la salud, atravesadas por situaciones de violencia de género intrafamiliar y por la mirada sancionatoria del sistema sanitario respecto a su función materna.

${ }^{23}$ PARELLA, Sonia. La mujer inmigrante: una triple discriminación. 
Y hay muchísimas mujeres bolivianas en situación de violencia (de género). Igual es muy difícil como te digo intervenir. No aceptan. Supongo que en parte tiene que ver con esto que te decía de los prejuicios porque esta cuestión de los hijos era sancionarlo como no los quiere, no le interesa... entonces verdaderamente si vos lo pensás yo tampoco me acercaría si las instituciones me responden desde una sanción... con otra cultura (psicóloga, grupo focal, mayo, 2016).

Los relatos del equipo de salud interdisciplinario ponen de manifiesto también la importancia de abordar la salud de las mujeres desde una aproximación que reconozca el contexto más amplio donde transitan las mujeres y generan sus prácticas en salud. En particular, se trata de comprender que la salud de las mujeres migrantes está signada además de por determinantes de género por aquellos de clase social y étnicos, lo cual refleja el carácter heterogéneo de la dimensión de género y la necesidad de pensar a la "mujer migrante" en relación a otras dimensiones sociales. La interrelación dinámica entre el género, la etnia y la clase social permite comprender los procesos de construcción de desigualdades sociales, dando cuenta que las modificaciones en estos procesos demandan transformaciones que involucren aspectos estructurales más amplios. En el relato siguiente se observa cómo la recuperación de las trayectorias de las mujeres permite a los profesionales abordar la salud individual desde una aproximación que recupera el valor colectivo y espiritual como forma para entender los padecimientos de las mujeres así como para proponer estrategias de atención donde "poder generar los dispositivos más adecuados para que puedan ellas encontrar la manera de transmitir...donde la palabra no sea el centro". Así se busca recuperar las trayectorias de vida de las mujeres, su rol dentro del proceso productivo, el valor comunitario de sus prácticas -como el tejido- y su forma de entender que "hay malestares que no sólo son físicos sino más espirituales" permite a los profesionales romper con las distancias que las separa de las mujeres y comenzar a establecer una relación médico-paciente desde una lógica horizontal.

Hay que simbolizar algo del cuerpo en que puede estar diciendo otras cosas. Hay malestares que no sólo son físicos sino más espirituales... siempre hay un en choque con la medicina, hay una gran limitación desde dónde se las escucha, entonces en el hacer ellos encuentran más, yo he visto que se engancha un montón más que si le propones el espacio de la palabra. Paulina (una paciente boliviana) nos hablaba que sabía tejer y le empezamos a llevar para tejer y eso permitió que empiece a hablar porque el tejido es importante en la cultura boliviana porque para ella tenía un enlazamiento con su país de origen (psicóloga, grupo focal, mayo, 2016). 


\section{El discurso epidemiológico del "riesgo" en el disciplinamiento de mujeres migrantes bolivianas}

A partir del análisis de discursos y prácticas de un agente sanitario del primer nivel de atención ${ }^{24}$ del departamento de San Carlos, Mendoza, esta sección muestra cómo el sistema biomédico se sirve de modo sustantivo de la epidemiología y de sus conceptualizaciones sobre el "riesgo" para fundamentar la manera en que se entienden los procesos de salud en el marco de la división sexual y social del trabajo de las mujeres migrantes bolivianas y los abordajes que se utiliza tanto preventivos como curativos. Nos interesa focalizarnos en la atención del agente sanitario que recorre un asentamiento rural de viviendas con población boliviana ubicado precariamente al costado de una ruta nacional para mostrar el rol que éste cumple en la construcción de mecanismos de regulación que operan sobre la forma de conceptualizar a la mujer migrante y a sus prácticas de salud. Nuestra intención es poner en evidencia distintos "mecanismos disciplinarios" que los agentes sanitarios despliegan para "adecuar" a las mujeres migrantes dentro de modelos de mujer y de salud más acordes a los parámetros esperados por el sistema de salud biomédico argentino. En este proceso, decimos, la gestión del agente sanitario pasa por delimitar el grupo social que conforman las mujeres bolivianas y establecer sus fronteras en el continuum de la población ${ }^{25}$, por fuera de las condiciones histórico-objetivas de existencia vinculadas al trabajo productivo-reproductivo que ellas desarrollan en el proceso migratorio.

Desde el sistema de atención primaria de la salud, los agentes sanitarios son los encargados de recorrer las calles de las comunidades a las que son asignados y recolectar información guiados por formularios en los cuáles se consignan datos fundamentalmente demográficos y epidemiológicos. Dentro del registro, la detección de situaciones de riesgo para la salud en el discurso epidemiológico resulta un pilar fundamental para la construcción del discurso biomédico, especialmente en cómo este último opera sobre las mujeres migrantes. Los discursos sobre la salud -y más específicamente, sobre los riesgos para la salud-consisten en construcciones circunstanciales, de carácter normativo, inapelablemente vinculadas a otros intereses, dependiendo así de definiciones de lo que es el ser humano, el tipo de sociedad que se anhela y los modos de alcanzarla ${ }^{26}$. Desde aquí, la cultura de las mujeres y de los migrantes bolivianos en general es conceptualizada de forma al menos dudosa,

\footnotetext{
${ }^{24}$ El primer nivel es la puerta de entrada al sistema de salud en tanto es el más cercano a la población encargado de la promoción de salud y prevención de la enfermedad donde se encuentran los agentes sanitarios que están distribuidos en áreas operativas comunitarias y realizan acciones de promoción, prevención y atención de la salud, especialmente materno-infantil.

25 FOUCAULT, op. cit.

${ }^{26}$ ALMEIDA FILHO, Naomar. Riesgo: concepto básico de la epidemiología, p. 323.
} 
confundida con las condiciones materiales de vida, y siempre como un aspecto negativo que se erige como una barrera para el acceso a la salud, la cual debe ser transformada.

De a poco (las mujeres) se están acostumbrando al ritmo del sistema de salud pero cuesta mucho. Muchas veces le tenés que decir "mirá mamá, decile a tu marido que no podés trabajar porque ya vas a tener (el bebé)", pero es cultural, ¿̇y qué podés hacer culturalmente?, hay otras culturas que digamos, nacieron así... qué sé yo, hay cosas que no las vamos a poder cambiar (agente sanitario, entrevista realizada en abril, 2016).

El discurso sobre el riesgo se manifiesta con particular énfasis en los casos donde existen mayores capas de subalternidad como es el caso de las mujeres migrantes bolivianas. En particular, surge del análisis que los supuestos sobre los que se construye el "riesgo" para la vida de las migrantes conlleva un entramado de prácticas de disciplinamiento y control orientadas a encauzar a estas mujeres en un determinado tipo de paciente y de mujer desde un triple sesgo de género, étnico-racial y de clase. Por un lado, el discurso del riesgo se erige desde una mirada acusatoria respecto a los comportamientos de las mujeres ante el cuidado de su salud y la de sus hijos que desconoce las realidades objetivas donde llevan adelante sus vidas en el contexto migratorio, las cuales dan lugar a estrategias y prácticas que se desarrollan en función de las condiciones de trabajo que supone la cosecha estacional.

Le tenés que hacer tomar conciencia que es un hijo y tiene una responsabilidad... porque están dando vueltas. Ahora se fueron a viajar un poquito más o se van... y no lo mandan (a los controles de salud), porque hasta que no termine (la cosecha) del tomate o del ajo es así (agente sanitario, entrevista realizada en abril, 2016).

Los estándares sobre la forma de cuidar la salud que deja entrever el agente sanitario, además de la reproducción de estereotipos de género vinculados a roles tradicionales de cuidado doméstico, tienen un importante sesgo normativo de clase que asume como dadas ciertas condiciones de vida por fuera de una historicidad y ajenas a las relaciones sociales que las mujeres despliegan en el contexto migratorio (por ejemplo, poder prescindir de una jornada laboral o disponer de permisos laborales para realizar consultas médicas). Hay una opinión formada sobre el rol que se espera que cumplan las mujeres bolivianas en el cuidado de su propia salud, pero sobre todo en el cuidado de quienes tienen a cargo y son desaprobadas ciertas acciones y estrategias que se dan en un marco de conciliación entre el cuidado y el trabajo remunerado.

Yo tendría un hogar lleno de niñitos porque encontrás niños solos todo el tiempo, todo el tiempo vas a encontrar niñitos de uno, dos o tres años al cuidado de una de doce... y le hacen de comer y todo así (agente sanitario, entrevista realizada en abril, 2016). 
La forma en que opera el discurso del riesgo en la reproducción de roles de género debe ser a su vez entendida en un marco más amplio caracterizado por un importante carácter eminentemente discriminatorio desde donde se erige el rol del agente sanitario como responsable natural de prevenir a la población nativa ante el potencial peligro que pueden tener los extranjeros, especialmente los de origen boliviano. Entender cómo se construyen desde el punto de vista de la "regulación de población (boliviana)" nos remite a los procesos de normalización que los mismos suponen. En este contexto, regular la familia boliviana -y especialmente a las mujeres como principales intermediarias "naturales" entre el sector salud y la unidad familiar-, supone desarrollar una serie de estrategias de control poblacional que operan como "normalizadoras" como una salvaguarda de la salud pública y del organismo político en tanto cuerpo. La cita siguiente pone de manifiesto cómo la mujer es colocada como responsable de controlarse y controlar a su familia potencialmente sospechosa para evitar la diseminación de una potencial enfermedad.

Vos (por él) tenés que tener la responsabilidad de ir, yo derivo tal mujer al hospital a algún lugar por falta de control de niño sano, por falta de control de embarazo, por sospechoso de una tuberculosis (agente sanitario, entrevista realizada en abril, 2016).

\section{Reflexiones finales}

A partir del análisis de las representaciones y prácticas de proveedores de salud del sistema sanitario de San Carlos y Tupungato, Mendoza, el artículo analiza cómo el sistema biomédico opera sobre la salud de las mujeres migrantes bolivianas, la construcción que se hace sobre el rol de las mujeres en el marco de la división sexual y social del trabajo así como su particular perspectiva de análisis y atención de los procesos de salud como procesos que construyen una forma de entender quién es el otro(a). El trabajo muestra distintos tipos de abordajes en la atención de las mujeres migrantes que varían entre aquellas donde se recuperan las condiciones objetivas e históricas donde las mujeres desarrollan sus procesos de salud y los distintos factores culturales, laborales y de género que las atraviesan en el contexto migratorio hacia otros donde se reproducen prácticas de disciplinamiento y control. La reconstrucción de las representaciones y prácticas del agente sanitario respecto a la salud de las mujeres migrantes bolivianas desde una perspectiva que contempla las diversas clasificaciones sociales interseccionalmente, vislumbra que el sistema de salud biomédico si bien se expresa como dominante ante todos los sistemas de atención y conjuntos sociales, lo hace con particular énfasis en los casos donde existen mayores capas de subalternidad como es el caso de las mujeres migrantes bolivianas a partir de su condición de género, étnico-racial, de nacionalidad y de clase social. Estas categorías operan como expresiones de poder y opresión 
donde, como mostramos con el caso del agente sanitario, el sistema biomédico se posiciona para ejercer su dominación frente a las migrantes. Desde aquí se crean y reproducen estereotipos de mujeres especialmente vinculados a los roles que ellas deberían seguir en su espacio privado como cuidadoras de su salud y la de sus hijos, como formas a su vez de proteger a la población nativa de un potencial peligro. Sin embargo, estos modos de atención conviven con otros como aquel que evidenciamos con el caso del equipo interdisciplinario en donde se cuestiona el sistema biomédico así como los sesgos que lo caracterizan y se incorpora a las necesidades de las mujeres en el abordaje sanitario. Desde aquí afirmamos que para comprender los procesos de salud de las mujeres migrantes bolivianas resulta clave historizar no solamente la reconstrucción de síntomas a lo largo del tiempo -rol fundamental de la historia clínica actual en el ámbito de la salud-, sino las trayectorias reproductivasproductivas que desarrollan en el contexto migratorio. Este trabajo muestra que cuando se construyen relaciones horizontales y de apertura para entender las trayectorias de las migrantes en el marco de un entramado de desigualdades interseccionales, mejora tanto el registro respecto a las problemáticas que atraviesan la salud de las mujeres como las condiciones para que ellas puedan romper con las resistencias respecto al uso del sistema sanitario. Desde esta perspectiva, lo intercultural no emana sólo a partir de un reconocimiento de la cultura de las mujeres bolivianas sino de un abordaje colectivo del proceso de salud que recupera las trayectorias laborales y de género y propone acciones que apuntan a incorporar a las mujeres migrantes, sus miradas y prácticas en los abordajes sanitarios.

\section{Referencias bibliográficas}

AIZENBERG, Lila; RODRíGUEZ, María Laura; CARBONETTI, Adrián. Percepciones de los equipos de salud en torno a las mujeres migrantes bolivianas y peruanas en la ciudad de Córdoba. Migraciones Internacionales, v. 8, n. 1, 2015, p. 65-94.

AIZENBERG, Lila. Hacía una aproximación crítica a la salud intercultural. Revista Latinoamericana de Población, v. 5, n. 9, 2011, p. 49-69.

ALMEIDA FILHO, Naomar. Riesgo: concepto básico de la epidemiología. Salud Colectiva, v. 5, n. 3, 2009, p. 323-344.

ANTHIAS, Floya. Belongings in a globalising and unequal world: rethinking translocations. In YUVAL-DAVIS, Nira; KANNABIRAN, Kalpana; VIETEN, Ulrike (eds.). The situated politics of belonging. London: Sage, 2006, p. 17-31.

AYALA, Encarnación. La mujer en la perspectiva intercultural. Madrid: Editorial La Muralla, 2006.

BAEZA, Brígida. La memoria migrante y la escucha de los silencios en la experiencia del parto de mujeres migrantes bolivianas en Comodoro Rivadavia (Chubut, Argentina). Anuario Americanista Europeo, n. 11, 2014, p. 179-197. 
BALÁN, Jorge. La economía doméstica y las diferencias entre los sexos en las migraciones internacionales: un estudio sobre el caso de los bolivianos en Argentina. Estudios Migratorios Latinoamericanos, v. 15, 1990, p. 269-295.

BENERIA, Lourdes. Trabajo productivo/reproductivo, pobreza y políticas de conciliación. Nómades, n. 24, 2006, p. 8-21.

CAGGIANO, Sergio. 'Que se haga cargo su país': la cultura, los Estados y el acceso a la salud de los inmigrantes bolivianos en Jujuy. In GARCÍA VÁZQUEZ, Cristina (ed.). Hegemonía e interculturalidad. Poblaciones originarias e inmigrantes. Buenos Aires: Prometeo, 2008, p. 243-279.

CARAMÉS GARCÍA, Mayte. Proceso socializador en ciencias de la salud. Caracterización crítica del modelo hegemónico vigente. In FERNÁNDEZ JUÁREZ, Gerardo (ed.). Salud e interculturalidad en América Latina. Perspectivas antropológicas. Quito: Abya Yala, 2004, p. 35-54.

CERRUTTI, Marcela. Diagnóstico de las poblaciones de inmigrantes en la Argentina. Serie de Documentos de la Dirección Nacional de Población, n. 02. Buenos Aires: Dirección Nacional de Población, Secretaría del Interior, 2009.

CERRUTTI, Marcela. Salud y migración internacional: mujeres bolivianas en la Argentina. Buenos Aires: PNUD- CENEP/UNFPA, 2011.

ESTEBAN, María Luz. El Estudio de la Salud y el Género: Las Ventajas de un Enfoque Antropológico y Feminista. Salud Colectiva, v. 2, n. 1, 2006, p. 9-20.

FOUCAULT, Michel. Vigilar y Castigar: Nacimiento de la prisión. Buenos Aires: Siglo XXI Editores Argentina, 2002.

GOOD, Bayron. Medicina, Racionalidad y experiencia: una perspectiva antropológica. Barcelona: Edicions Belaterra, 2003.

KLEINMAN, Arthur. Writing at the margin. Discourse between anthropology and medicine. Berkeley: University of California Press, 1995.

MARIANO, Lorenzo. Culturas biomédicas. Textualidades y narrativas opacas en la atención a la población inmigrante. In FERNÁNDEZ JUAREZ, Gerardo (ed.). La Diversidad frente al espejo. Salud, interculturalidad y contexto migratorio. Quito: Abya Yala, 2008, p. 305-320.

MARTÍNEZ PIZARRO, Jorge; REBOIRAS-FINARD, Leonardo. Migración, derechos humanos y salud sexual y reproductiva: delicada ecuación en las fronteras. Pap. Poblac, v. 16, n. 64, 2010, p. 9-29.

$\mathrm{NASH}$, Mary. Identidad cultural de género, discurso de la domesticidad y la definición del trabajo de las mujeres en la España del siglo XIX. In DUBY, Georges; PERROT, Michelle (dir.). Historia de las mujeres. El siglo XIX. Madrid: Taurus, Tomo IV, 1993, p. 585-597.

PANTELIDES, Edith; MORENO, Martín. Situación de la población en Argentina. Buenos Aires: PNUD/UNFPA, 2009.

PARELLA, Sonia. La mujer inmigrante: una triple discriminación. Barcelona: Anthropos, 2003. 
PESSAR Patricia; Sarah MAHLER. Transnational Migration: Bringing Gender. International Migration Review, v. 37, n. 3, 2003, p. 812-846.

RODRÍGUEZ, Lilia. Factores Sociales y Culturales Determinantes en Salud: La Cultura como una Fuerza para Incidir en Cambios en Politicas de Salud Sexual y Reproductiva. Ponencia presentada en el III Congresso da Associação Latino Americana de População, ALAP. Córdoba, 2008.

SASSEN, Saskia. Globalization and its Discontents: Essays on the New Mobility of People and Money. New York: The New Press, 1998.

Recibido para publicación en 31.01.2017

Aceptado para publicación en 25.09.2017

Received for publication in January $31^{\text {st }}, 2017$

Accepted for publication in September 25

ISSN impresso 1980-8585

ISSN eletrônico 2237-9843

http://dx.doi.org/10.1590/1980-85852503880005110 